\title{
DESARROLLO DE UN GENERADOR DE GASES AUTOMÁTICO CONTROLADO POR PLC
}

\author{
Javier Díaz-Pajuelo, Jesús Lozano, José Ignacio Suárez, Patricia Arroyo, Antonio José Calderón \\ Escuela de Ingenierías Industriales, Universidad de Extremadura \\ Av. Elvas s/n. Badajoz. Email: jesuslozano@unex.es
}

\begin{abstract}
Resumen
Se propone en esta comunicación un sistema generador de gases de automático mediante el cual generar una concentración conocida de hasta cuatro gases o una mezcla de ellos con el objetivo de calibrar cualquier tipo de sensores o detectores de gases. El sistema consta de cuatro entradas controladas por cuatro caudalímetros y cuatro electroválvulas, un módulo de expansión de gases, medidas de seguridad para finalizar el proceso en caso de fallo y por último, un armario robusto que lo contiene. El sistema de control está basado en un autómata V-350 de Unitronics con sistema SCADA incluido mediante el cual controlar el proceso y dos módulos de expansión. Se han realizado una serie de pruebas con objeto de validar el sistema. Para ello se han generado diferentes gases en diferentes concentraciones, obteniendo resultados satisfactorios.
\end{abstract}

Palabras Clave: Control de proceso, controladores lógico programables, control de caudales.

\section{INTRODUCCIÓN}

Tres cuartas partes de la población europea viven en áreas urbanas, en las que se lleva a cabo una intensa actividad económica e industrial que causa una importante parte de la contaminación ambiental del planeta. Dicha contaminación tiene efectos perniciosos en la salud de los seres vivos. Para revertir esta tendencia y reducir la contaminación, los gobiernos están estableciendo normativas medioambientales cada vez más estrictas que obligan a la monitorización constante de la calidad del aire en múltiples ubicaciones.

La instrumentación empleada en la medida de la contaminación atmosférica es muy variada, y va desde aparatos como los Lidar [1] (radares láser), que pueden alcanzar un coste de varios millones de euros y ocupar un camión, a sensores de estado sólido de unos pocos euros y que caben en la palma de la mano. Estos instrumentos se puede clasificar en:
- Sensores y analizadores, que proporcionan una medida de la concentración de contaminantes. Son los más interesantes desde el punto de vista de la tecnología medioambiental.

- Detectores, que solamente dan una indicación de umbral, como los detectores de gases tóxicos o explosivos, en los que una indicación de alarma se dispara por encima de un nivel prefijado.

Por otra parte, la distinción entre sensores y analizadores es menos definida, pero suele atribuirse a los sensores las siguientes características:

- Posibilidad de utilizarse en régimen continuo o casi continuo, es decir, sin tener que tomar muestras discretas.

- No utilizan reactivos químicos

- No interviene un operario en cada medida para la toma de muestras, calibración, etc.

En este sentido, los sensores químicos tienen hoy en día un interés renovado debido a las necesidades actuales de disponer de más y mejor información en tiempo real además de las ventajas que supone su utilización frente a los grandes equipos analíticos, de elevado coste, de manipulación especializada y confinados en recintos acondicionados. En este sentido, los sensores químicos representan una nueva clase de instrumentación analítica, caracterizada por unas pequeñas dimensiones, un bajo coste, una utilización amigable y una generación de la información (idealmente) en tiempo real [3].

Un sensor químico se puede definir como un dispositivo que transforma información química en una señal analítica útil. Consta, principalmente, de dos partes: un receptor, que proporciona el reconocimiento de la sustancia a analizar, y un transductor, que convierte la señal química obtenida de la sustancia en una señal medible por un instrumento. El principal problema de los sensores químicos es la falta de especificidad a un único gas y en ocasiones se ven afectados por otros gases diferentes al que se pretende medir, además de la humedad y la temperatura, lo cual genera errores en la medida con estos dispositivos. Para evitar estos errores, es necesaria la calibración de estos sensores 
para compensar efectos no deseados por otros compuestos y ofrecer una medida correcta frente al gas objetivo.

Con este objetivo, se presenta en este trabajo un sistema generador de gases de automático mediante el cual generar una concentración conocida de hasta cuatro gases o una mezcla de ellos con el objetivo de calibrar cualquier tipo de sensores o detectores de gases. En el siguiente apartado se describirá de forma breve el sistema desarrollado, incluyendo las diferentes partes y los controladores utilizados. En la sección 3 se muestra el programa de control diseñado para el gobierno del sistema de forma automática y por último en el apartado 4 se presentan una serie de medidas realizadas para la validación del sistema.

\section{DESCRIPCIÓN DEL SISTEMA.}

Es un sistema generador de gases de control automático, controlado mediante un sistema SCADA, con 4 puertos de entrada de gases y uno de salida, que se conectará al detector de gases a calibrar. Todo ello tiene un diseño robusto al estar contenido en un armario diseñado a medida para soportar un ambiente industrial, con medidas de seguridad que permiten parar el proceso de generación de gases en caso de pulsar el botón por un posible fallo en el sistema. También se dispone de comunicaciones de tipo serie para poder manejar el equipo de forma remota con un ordenador.

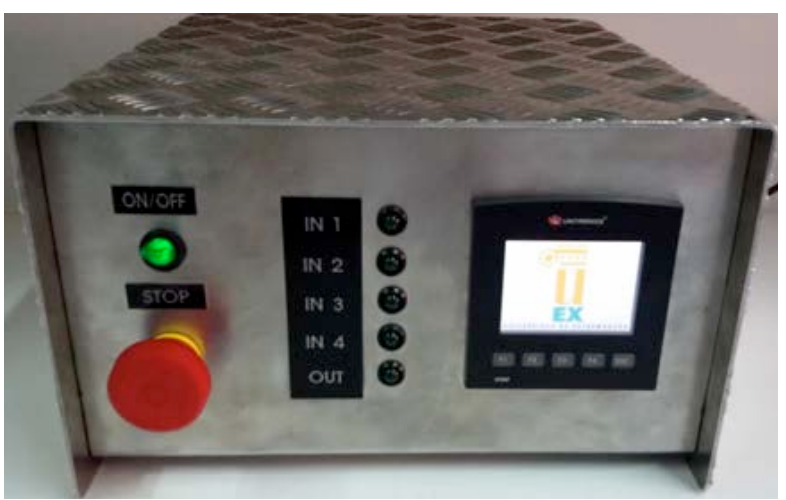

Figura 1: Generador de gases desarrollado.

\subsection{PARTES}

Para la realización del sistema de generación de gases automático se han utilizado las siguientes piezas o dispositivos:

- Caudalímetros.

- Electroválvulas.

- PLC.

- Módulos de expansión del PLC.

- Módulo de expansión de gases.
- Armario metálico

\subsection{CAUDALÍMETROS}

Los caudalímetros con los que se ha trabajado han sido de la marca Brooks, más concretamente de la serie 5800-s. Son unos caudalímetros másicos térmicos y se comunican con el autómata programable mediante un conector de 14 pines [4]. En la Figura 2 se muestra una tabla con la distribución de señales del conector y señalados aquellos que se han utilizado en la aplicación.

\begin{tabular}{|c|c|}
\hline Pin (TMF side) & Function \\
\hline 1. & Setpoint return (-) \\
\hline 2. & $0(1)-5$ Vdc Flow signal output \\
\hline 3. & $(\mathrm{TTL})$ Open collector alarm output \\
\hline 4. & $0(4)-20$ mA Flow signal output \\
\hline 5. & $+15 \mathrm{Vdc}$ Power supply \\
\hline 6. & $-15 \mathrm{Vdc}$ Power supply (if required) \\
\hline 7. & $0(4)-20$ mA Setpoint input (+) \\
\hline 8. & $0(1)-5$ Vdc Setpoint input $(+)$ \\
\hline 9. & Power supply common \\
\hline 10. & Flow signal output common \\
\hline 11. & +5 Vdc ref. output \\
\hline 12. & Valve override input \\
\hline 13. & Not connected \\
\hline 14. & Optional RS232C-RxD or RS485-A- \\
\hline
\end{tabular}

Figura 2: Conector de comunicación del caudalímetro.

Se han utilizado 4, dos de ellos con rangos de caudal, de 0 a $10 \mathrm{~mL} / \mathrm{min}$ de caudal para medidas con más precisión y otros dos de 0 a $100 \mathrm{~mL} / \mathrm{min}$. Los cuales se alimentan a una tensión de $15 \mathrm{Vcc}$ y se comunican con el exterior mediante diferencias de tensión de entre 0 y $5 \mathrm{~V}$.

\subsection{PLC Y MÓDULOS DE EXPANSIÓN}

El PLC escogido ha sido un V-350 de la marca Unitronics con las siguientes características [5]:

- Pantalla táctil de 3,5 ".

- 12 entradas digitales y 2 analógicas.

- 8 salidas por relés y 2 analógicas.

- Tiempo de escaneo de $15 \mu$ ser por cada 1 $\mathrm{Kb}$.

- Tarjeta micro SD.

- 1 puerto RS232.

Además de este PLC se ha tenido que adquirir otro módulo de entradas y salidas analógicas ya que se necesitan 4 entradas y salidas analógicas, por ello, se ha elegido el módulo de expansión IO-AI4-AO2 el cual trae consigo cuatro entradas y dos salidas analógicas, ambas pueden controlarse, tanto variando la tensión de 0 a $10 \mathrm{Vcc}$ como de corriente, de 0 a 20 $\mathrm{mA}$, al igual que ocurre con las entradas y salidas del PLC V-350. 


\subsection{ELECTROVÁLVULAS}

Las electroválvulas escogidas fueron unas Joucomatic de $24 \mathrm{Vcc}$ para el cierre hermético de cada una de las líneas del circuito neumático ya que los caudalímetros no pueden proporcionar un cierre completo.

\subsection{MÓDULO DE EXPANSIÓN DE GASES}

Está formado por un cilindro de $3 \mathrm{~cm}$ de diámetro unido por sus extremos al circuito, con dos finalidades: primera, para absorber los cambios de presión producidos al abrir y cerrar las distintas válvulas, y en segundo lugar, al producirse también una depresión al aumentar el diámetro del conducto, mejorar la homogeneidad de la mezcla creada.

\subsection{CIRCUITOS DEL SISTEMA}

Con objeto de describir el funcionamiento del dispositivo desarrollado, se dividirá en tres partes, desde un punto de vista funcional: circuito de gas, circuito de control y armario.

\section{Circuito de gas}

Encargado de generar la mezcla de gases objetivo para la calibración. El proceso comenzará en la parte posterior del armario en el cual se encuentran situadas en las cuatro entradas de gas que irán conectadas a cuatro botellas de diferentes gases. Estas, estarán localizadas debajo de una chapa en la cual están grabados los números de las entradas.

Las entradas serán 4 apliques neumáticos pasamuros, los cuales conectaran seguidamente con cada uno de sus caudalímetros respectivos, y a continuación con sus electroválvulas respectivas. Estos cuatro circuitos de gas seguirán en todo momento una trayectoria paralela, como se puede observar de forma esquemática en la Figura 3a y una imagen real en la $3 \mathrm{~b}$.

A la salida de las electroválvulas y mediante racores de "codo" y "T" se unirán todos en un mismo conducto el cual ira en perpendicular a los otros cuatro y mediante otro codo metálico se conectara al módulo de expansión de gases que estará colocado en paralelo con las entradas.

Por último, del módulo de expansión de gases se conectará a la salida. La mezcla saldrá del armario por su parte posterior mediante un aplique metálico pasamuros neumático, e irá conectado a la celda de sensores que se pretende calibrar. Justo encima de la salida está situada una chapa con la inscripción "out" grabada.

\section{CIRCUITO DE GAS}

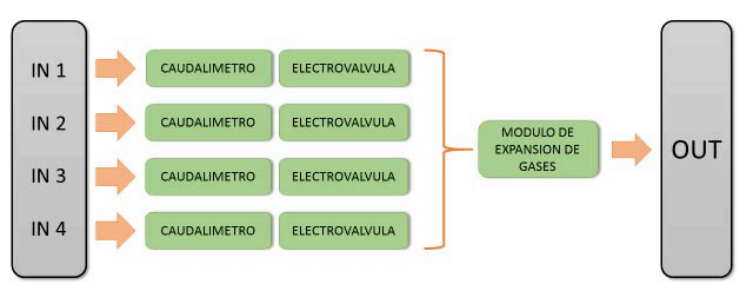

a)

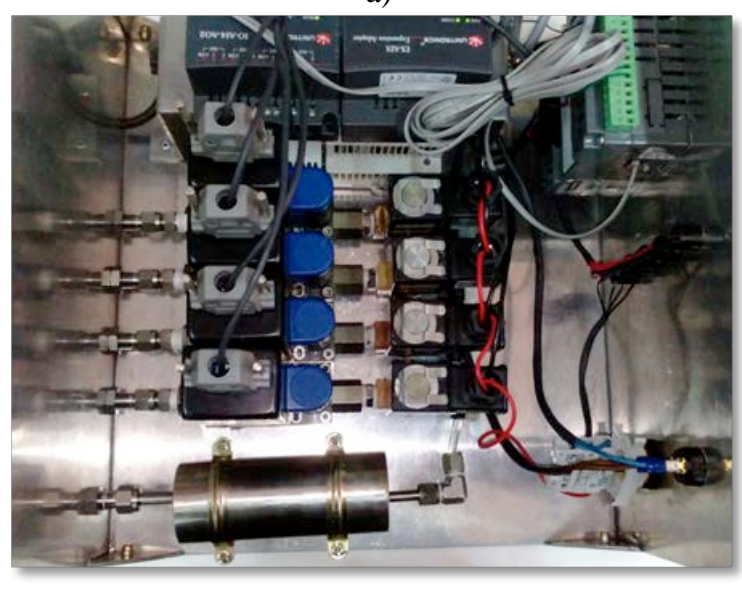

b)

Figura 3: Circuito de gas.

\section{Circuito de control}

Tal y como se ha comentado, se va a realizar un control basado en la utilización de un PLC junto con varios circuitos y conexiones diseñadas y construidas para este prototipo. En la Figura 4 se muestra el circuito de control.

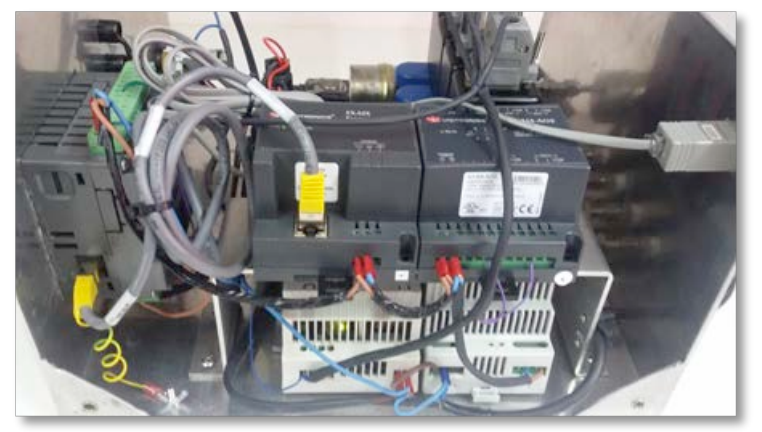

Figura 4: Circuito de control.

En la parte central se encuentran colocadas en dos niveles y mediante carriles DIN los dispositivos de control. En el nivel inferior, dos fuentes de alimentación, de 24 y $15 \mathrm{~V}$ respectivamente. La fuente de $24 \mathrm{~V}$ alimentara al PLC, a sus módulos de expansión, a las electroválvulas y por último los indicadores LED y la de $15 \mathrm{~V}$ a los caudalímetros. En el nivel superior se encuentran, el PLC junto con sus módulos de expansión y están conectados tanto por sus entradas como por sus salidas a los caudalímetro 
y se comunican con ellos mediante señales analógicas. A los pines de salida del PLC están conectados también tanto los indicadores LED como las Electroválvulas, estas últimas conectadas a unas salidas de relés.

El control de la concentración de cada gas es realizado por cada caudalímetro, limitándose el PLC a sacar por los puertos de salida analógico las tensiones correspondientes al caudal seleccionado.

\section{Armario}

En su cara frontal (Figura 1) se encuentra un interruptor mediante el cual cortar la corriente del sistema al completo, un botón de emergencia o seta para cortar la corriente solo a los indicadores LED y las electroválvulas, ya que así, si surge cualquier imprevisto se podría parar el proceso sin necesidad de cortar la corriente al sistema completo. A su derecha se encuentran 5 Indicadores Led con la función de mostrar el estado de las entradas y salida. Por último un sistema SCADA que permite gobernar y monitorizar el proceso mediante una serie de pantallas que se explicarán posteriormente. En su cara posterior se encuentran las entradas y salida del gas y un conector RS232 mediante el cual controlar el sistema desde un ordenador ajeno al proceso.

\section{DISEÑO DEL PROGRAMA DE CONTROL}

El diseño y funcionamiento del programa está ideado para que una persona sin conocimientos técnicos del sistema sea capaz de trabajar con él. La programación se ha realizado mediante el software Visilogic [6] que es el de programación del PLC elegido y se ha dividido en dos:

- Programación del PLC.

- Programación del SCADA.

\subsection{PROGRAMACION DEL PLC}

Para la programación del PLC se ha utilizado esquema de contactos o Ladder y para ello se han utilizado además de los contactos, bloques de todo tipo desde matemáticos, temporizaciones, guardado de tablas en tarjeta SD, etc. Un ejemplo de esta programación se muestra a continuación: la apertura y cierra de una electroválvula mediante una botonera. En ella si se presiona el botón "F1" y la electroválvula está abierta, se cerrara, se apagara el indicador LED y se reiniciara el temporizador de esa línea del proceso, en el caso contrario se abrirá la electroválvula y se encenderá el indicador LED.

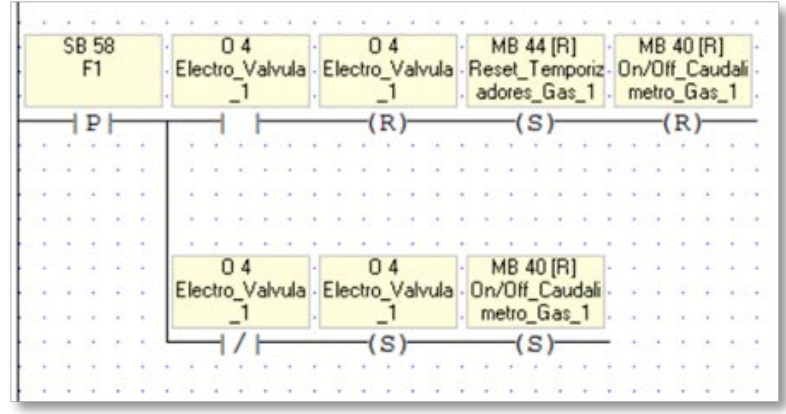

Figura 5: Ejemplo de programación del PLC.

\subsection{PROGRAMACION DEL SCADA}

Para hacer más sencillo el manejo del generador de gases se ha utilizado una Interfaz Hombre Máquina basado en SCADA en la que se utilizan una serie de pantallas que se describen a continuación:

- Presentación

- Menú de inicio

- Gases principales

- Elección de proceso

- Programación de proceso

- Descripción de proceso

- Graficas del proceso en tiempo real

- Valor actual de entradas en tiempo real

\section{Presentación y Menú de inicio}

Al encender el sistema se inicia con la pantalla de presentación, la cual se mantiene durante 5 segundos y pasa automáticamente a la página de menú de inicio. Esta, está formada por tres botones, "Automático" el cual nos dirige a la pantalla elección de procesos guardados, "Manual", dedicado para casos en los que no haga falta guardar la configuración y el botón de "Gases principales", el cual dirigirá a la pantalla con su mismo nombre.

\section{Gases principales}

Está Dedicada a la edición de las entradas; esto es necesario, ya que, los caudalímetros han sido calibrados para $\mathrm{N}_{2}$, por lo tanto, al utilizarse gases de diferente densidad, hay que aplicar a los resultados un factor de corrección que se puede especificar en esta pantalla además de los nombres de los gases a mezclar.

\section{Elección de proceso}

En la pantalla de elección de procesos, se puede elegir entre 4 procesos o mezclas de gases guardados con anterioridad. Cada uno de ellos dirigirá al usuario a la pantalla de programación del proceso correspondiente. 


\section{Programación del proceso}

Esta pantalla, mostrada en la Figura 6, está dividida en dos partes claramente diferenciadas. A la izquierda se encuentran cuatro tablas en las cuales se puede definir el caudal de flujo deseado y durante cuánto tiempo se desea.

A la derecha se encuentra una botonera la cual permite retroceder a la pantalla anterior, ver los valores de entrada actuales, ver las gráficas en tiempo real, editar la descripción del proceso y por último, poner en marcha el proceso.

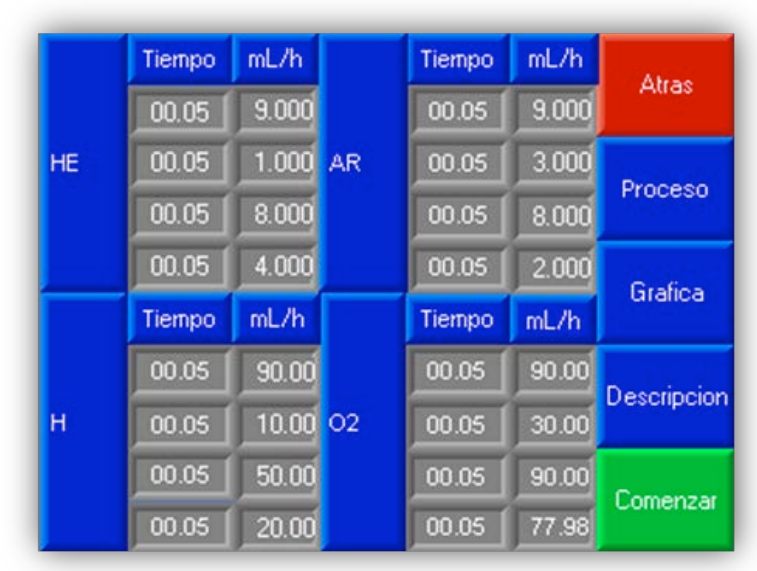

Figura 6: Pantalla de programación del proceso.

\section{Descripción del proceso}

En ella se puede editar el nombre, el tiempo de duración del proceso y el porcentaje de cada gas contenido en la mezcla.

\section{Valores actuales de entrada}

En esta pantalla se pueden observar el valor de flujo deseado de cada gas frente al valor real de flujo.

\section{Grafica del proceso}

Esta pantalla es la que nos indica un histórico de los valores reales de cada uno de los caudalímetros así como el estado de electroválvulas y caudalímetros. En las Figuras 7 y 8 se muestra esta pantalla para las pruebas realizadas. En la parte superior se encuentran 8 indicadores los cuales nos indican el estado (abierto o cerrado) de las electroválvulas y caudalímetros. En zona central se encuentra la gráfica de representación de los valores de las cuatro entradas con respecto al tiempo y en la parte inferior se halla una botonera donde aumentar o disminuir el flujo de las entradas en tiempo real.

\section{RESULTADOS}

Con objeto de comprobar el correcto funcionamiento del sistema desarrollado, se han realizado las siguientes pruebas:

- Disolución de benceno en aire.

- Aumento escalonado del caudal de flujo de un gas.

- Apertura de las cuatro entradas al mismo tiempo.

\section{Disolución de benceno en aire}

En ella se ha realizado una disolución al $10 \%$ de benceno, para ello simplemente se debe tener en las entradas $10 \mathrm{~mL} / \mathrm{min}$ de benceno y $90 \mathrm{~mL} / \mathrm{min}$ de aire. Como se observa en la Figura 7, existe un régimen transitorio debido a la apertura de las electroválvulas en el que se produce una oscilación en la señal. Este estado se mantendrá durante un segundo y entra en el régimen estacionario donde las señales son totalmente estables, lo que permite obtener las concentraciones requeridas.

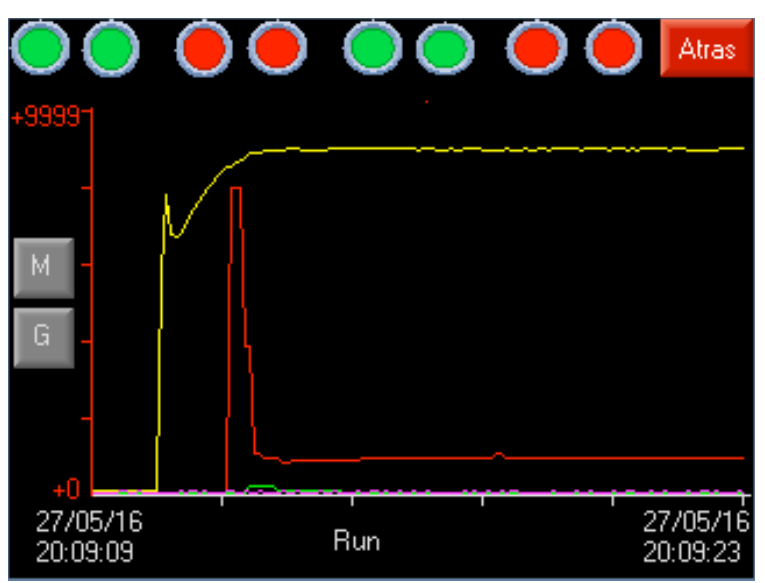

Figura 7: Disolución de benceno en aire.

\section{Aumento escalonado del caudal de flujo}

Como indica el apartado, se ha aumentado el caudal de flujo de 0 a $100 \mathrm{~mL} / \mathrm{min}$ en escalones de 25 $\mathrm{mL} / \mathrm{min}$ cada 2 segundos. Se pueden ver los valores de los caudales obtenidos en la Figura 8. Además se puede observar cómo tras un breve tiempo de estabilización, los caudales se ajustan a lo prefijado. 


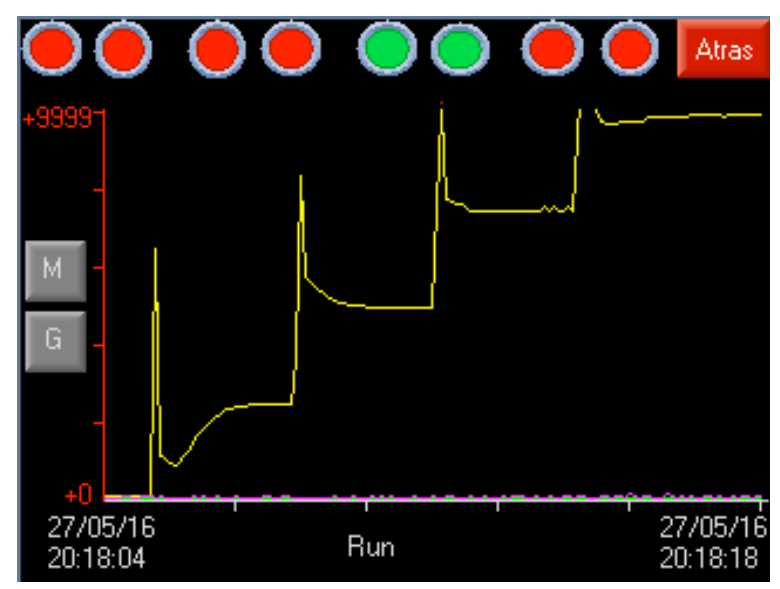

Figura 8: Aumento escalado del flujo.

\section{Apertura de las cuatro entradas al mismo tiempo}

Esta prueba se ha realizado para comprobar que excepto en el régimen transitorio, las entradas no se afectan las unas a las otras.

\section{CONCLUSIONES}

El equipo desarrollado y descrito en la presente comunicación ha cumplido los objetivos de diseño y especificaciones planteados y será de gran utilidad para la calibración de sensores y detectores de gases así como para la realización de medidas con estos dispositivos. El prototipo además incorpora medidas se protección de parada instantánea del proceso ante cualquier emergencia que pueda surgir (por ejemplo, escape de gases). Además el sistema permite su conexión a un PC desde el que se puede realizar la programación del mismo de forma más cómoda. Por último, se han completado las pruebas satisfactoriamente, cumpliendo con los valores de aceptación de las distintas medidas realizadas.

\section{Agradecimientos}

Este trabajo ha sido realizado gracias al apoyo del Ministerio de Economía y Competitividad a través del proyecto TEMINAIR (TEC2013-48147-C6-5).

\section{Referencias}

[1] Claus Weitkamp "Lidar: Range-Resolved Optical Remote Sensing of the Atmosphere", Springer Science \& Business. 2006.

[2] Ernesto Martínez Ataz, Yolanda Díaz de Mera Morales "Contaminación atmosférica" Univ de Castilla La Mancha, 2004 - 288 páginas

[3] Lorenzo Leija, "Métodos de procesamiento avanzado e inteligencia artificial en sistemas sensores y biosensores" Editorial Reverte, 2010

[4] Datasheet de los caudalímetros Brooks 5800. Disponible en (visitado el 20/06/2016): http://www.servinstrumentation.fr/commun/pdf/ en/E_Brooks_5850S_OK.pdf

[5] Características del PLC V-350 de Unitronics. Disponible en (visitado el 20/06/2016): http://unitronicsplc.com/vision-seriesvision $350 /$

[6] Software de programación Visilogic. Manual de Usuario. Disponible en (visitado el 20/06/2016): http://www.unitronics.com/Downloads/Support/ Technical\%20Library/VisiLogic\%20Software/S oftware\%20Manuals/VisiLogic\%20\%20Getting_Started.pdf 